\title{
Streaming video-based 3D reconstruction method compatible with existing monoscopic and stereoscopic endoscopy systems
}

\author{
Henri Bouma ${ }^{* a}$, Wannes van der Mark ${ }^{a}$, Pieter T. Eendebak ${ }^{\mathrm{a}}$, Sander H. Landsmeer ${ }^{\mathrm{a}}$, Adam W.M. \\ van Eekeren $^{\text {a }}$, Frank B. ter Haar ${ }^{a}$, F. Pieter Wieringa ${ }^{\text {a }}$, Jean-Paul van Basten ${ }^{b}$ \\ a TNO, PO Box 96864, 2509 JG The Hague, The Netherlands; \\ ${ }^{\mathrm{b}}$ Canisius Wilhelmina Hospital, PO Box 9015, 6500 GS Nijmegen, The Netherlands.
}

\begin{abstract}
Compared to open surgery, minimal invasive surgery offers reduced trauma and faster recovery. However, lack of direct view limits space perception. Stereo-endoscopy improves depth perception, but is still restricted to the direct endoscopic field-of-view. We describe a novel technology that reconstructs 3D-panoramas from endoscopic video streams providing a much wider cumulative overview. The method is compatible with any endoscope. We demonstrate that it is possible to generate photorealistic 3D-environments from mono- and stereoscopic endoscopy. The resulting 3D-reconstructions can be directly applied in simulators and e-learning. Extended to real-time processing, the method looks promising for telesurgery or other remote vision-guided tasks.
\end{abstract}

Keywords: Minimal invasive surgery, 3D reconstruction, endoscopy, e-learning, telemedicine.

\section{INTRODUCTION}

Compared to open surgery, minimal invasive surgery (MIS) offers many advantages, such as reduced trauma and scarring, less pain and complications, faster recovery (reconvalescence) and shorter hospitalization time. However, indirect viewing with an endoscope limits (anatomical) orientation and space perception during MIS. In quite a few surgical applications the endoscope is explicitly used to systematically scan a body cavity. Examples are: gastroscopy, colonoscopy, in uterus endoscopic treatment of twin-to-twin transfusion syndrome, as well as endoscopic treatment of bladder cancer and the periodical control cystoscopies over (a life-long period) 5 years thereafter, etc. Surgeons must build a mental 3D-model from sequentially scanned anatomical details. Stereo-endoscopy (e.g. using the Da Vinci robot [14] or hand-held (laparo) endoscopes [36]) improves depth perception, but still only offers stereopsis within the direct endoscopic field-of-view.

We describe a novel technology that can reconstruct 3D panoramas from video streams of endoscopically explored body cavities. This medical application builds further upon our previously described 3D crime-scene reconstruction technology [13]. The method is basically compatible with any endoscope and provides a much wider cumulative overview than single endoscopic image frames can offer. The technology is an add-on to existing endoscopy systems. It does not need position tracking, additional markers or expensive (MR or CT) pre-scans, because it is purely based on information that is extracted from the endoscopic images.

We demonstrate photorealistic 3D environments generated from single (mono) and dual channel (stereo) laparoscopic video data. Stereo endoscopes are becoming more-and-more standard tools for MIS [32]. Our 3D environments can be directly applied in simulators for endoscopic surgery and e-learning, showing 3D-views that respond to manipulation of a joystick or other endoscopic camera simulator tool. When practicing with an endoscopic simulator, the method can also visualize position and orientation of the simulated endoscope relative to the environment as viewed from a virtual perspective outside the simulated endoscope; improving the situational awareness of the operator and mentor. The method also allows measurement of distances and sizes. If extended to real-time processing, the method looks promising for tele-surgery or other remote vision-guided tasks requiring spatial visualization and complex navigation.

* henri.bouma@tno.nl; phone +31.88.866.4054; http://www.tno.nl

Henri Bouma, Wannes van der Mark, Pieter T. Eendebak, Sander H. Landsmeer, Adam W. M. van Eekeren, Frank B. ter Haar, F. Pieter Wieringa and Jean-Paul van Basten, "Streaming video-based 3D reconstruction method compatible with existing monoscopic and stereoscopic endoscopy systems", Proc. SPIE, Vol. 8371, 837112 (2012); http://dx.doi.org/10.1117/12.918592

Copyright 2012 Society of Photo-Optical Instrumentation Engineers (SPIE). One print or electronic copy may be made for personal use only. Systematic reproduction and distribution, duplication of any material in this paper for a fee or for commercial purposes, or modification of the content of the paper are prohibited. 
The further outline of this paper is as follows: We describe the method in the Sections 2 to 5. In the last sections we present our experiments and results (Section 6) followed by conclusions (Section 7).

\section{OVERVIEW OF THE METHOD}

Our 3D reconstruction approach consist of four main components, which are shown in Figure 1. Streaming video from the endoscope system forms the system input. The first component is motion estimation, which computes the endoscope's changes in position and orientation. This component is described in Section 3. Based on this information, the second component can reconstruct dense sets of visible surface points in 3D (Section 4.1). In the next component, the 3D points are converted into 3D surfaces (Section 4.2). Finally, texture mapping is applied for the 3D visualization of the result (Section 5).

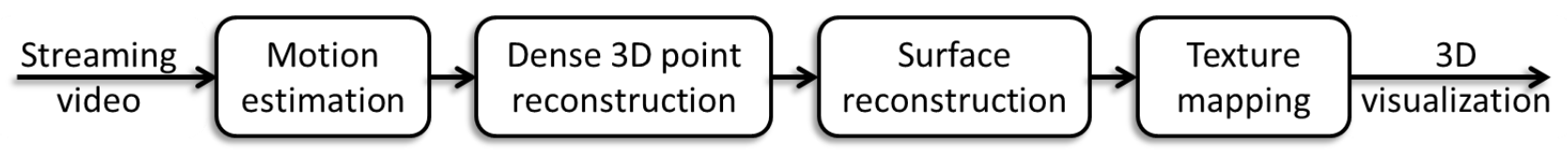

Figure 1. Main components of the 3D reconstruction method.

\section{MOTION ESTIMATION}

\subsection{Aided motion estimation}

Many aids have been proposed to assist motion estimation: position tracking [18][27], active imaging with structured light [6][19][23] or time of flight [29], additional markers [3] or expensive (MR or CT) pre-scans [5][12]. In contrast to these approaches, our method is purely based on information that is extracted from the existing endoscopic images.

\subsection{Monoscopic ego-motion estimation}

Monoscopic ego-motion estimation uses streaming video of a single moving camera to determine the camera position. The camera location is estimated in an unknown scene and simultaneously a map of the environment is created by simultaneous localization and mapping (SLAM) [7][9]. Several methods have been proposed to perform ego-motion estimation, such as Bundler [30], the PTAM framework [17] and the FIT3D toolbox [10]. In environments with rich textural information and an off-line scenario - where post-processing is allowed - Bundler resulted in a good ego-motion estimation and was therefore used in our monoscopic experiments.

\subsection{Stereoscopic ego-motion estimation}

Ego-motion can be estimated for a moving stereo endoscope with either monoscopic or specialized stereo-based methods. Monoscopic ego-motion estimation is based on the structure-from-motion paradigm; which means that the camera must always be moved first in order to obtain 3D information about its environment. However, during some surgical procedures it might not be possible or even desirable that the endoscope is extensively moved. In such cases, a stereo endoscope can be used in order to obtain 3D information by exploiting stereopsis. The advantage of the stereo approach is that the $3 \mathrm{D}$ model can be generated even when the endoscope is not moved.

We use the accurate and robust methods for ego-motion estimation developed by [8]. Real-time ego-motion estimation places each subsequent camera position in the global 3D coordinate system. This approach enables a real-time update of the 3D model.

\section{POINT AND SURFACE RECONSTRUCTION}

\subsection{Dense 3D point reconstruction}

During ego-motion estimation, only a sparse set of 3D points is obtained. There are several options to obtain a denser 3D point reconstruction (Figure 2). Strecha et al. give an excellent overview [31]. Conventionally, dense disparity estimation 
is applied between two images, recorded at different viewpoints. For an example of a stereo disparity algorithm that takes rectified stereo images from a calibrated set-up, see van der Mark et al. [21]. This approach assumes that the translation and orientation difference between the two viewpoints is exactly known, so that the 3D stereo reconstruction can be performed with the real world scale. However, for our experiments we have used two different approaches that can combine information from more than two viewpoints to obtain dense point clouds.

The first method is a multi-view approach as developed by Furukawa [11] and used in PMVS. Instead of using just two images - as in conventional stereo disparity estimates - as much images as possible are used to reconstruct 3D surface points. This procedure assumes that all camera positions have been computed before the dense reconstruction starts. The process starts with a sparse set of matched keypoints, and repeatedly expands these to nearby pixel correspondences before using visibility constraints to remove false matches.

The second method is similar to that of Newcombe [24][25]. It uses optical flow for the dense matching between pairs of images [34] and then computes the 3D position of points with stereo triangulation [15]. This approach results in highquality accurate reconstructions with only a low number of holes. The technique can be applied to left-right images of one stereo-pair and to monoscopic images over time. An advantage of the stereo approach is that we obtain depth values for many pixels in the left image and when the neighbor pixels are connected with triangles before placing the $2 \mathrm{D}$ pixels in the 3D world we easily acquire a triangular mesh for each stereo pair.

For visualization purposes the actual scale of the $3 \mathrm{D}$ reconstruction is less important, unless the final reconstruction is used for measurements. A calibration procedure is available to acquire the camera model and the relation between the left and right camera of the stereo-endoscope. In a fixed setup this calibration has to be performed only once. Once the stereo-endoscope is calibrated, the 3D stereo reconstruction acquires the real world scale.

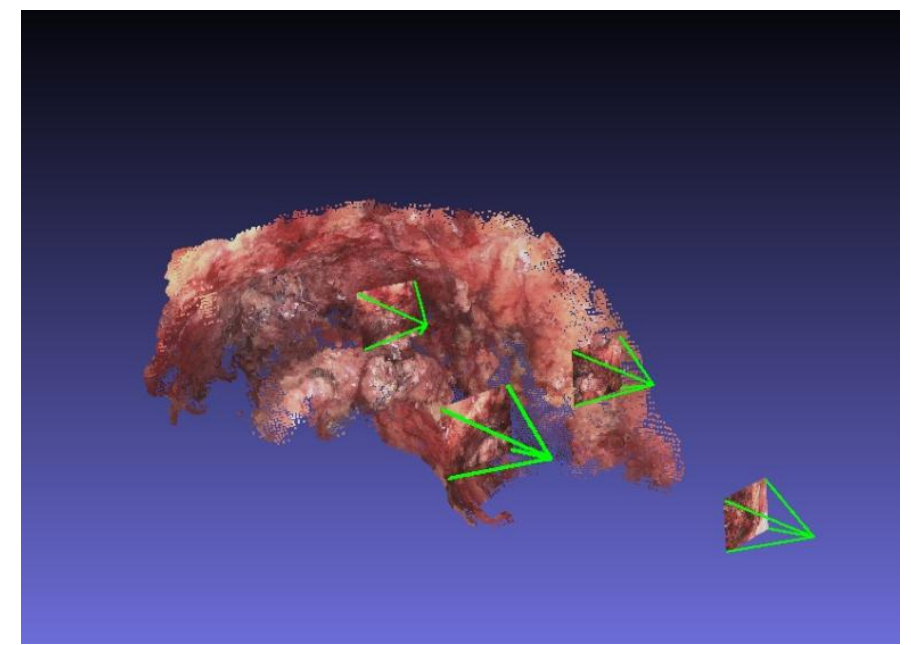

Figure 2. Reconstructed 3D points with examples of corresponding camera positions.

\subsection{Surface reconstruction}

This subsection describes the step from a dense 3D point cloud to a geometric model of a smooth surface mesh (Figure 3 ). This conversion allows us to separate the geometrical shape model and the textural color description. The separation enables fast creation of shape models at acceptable quality while preserving all details that are visible in the input highdefinition video stream.

Our dense stereoscopic method creates a dense surface for each stereo pair and uses the ego-motion estimation to align these surface meshes. To create a single surface mesh, we consider the fact that we either have a dense point cloud or a set of aligned surfaces meshes. For both types of 3D reconstructions we only consider the point clouds and fit a new surface mesh through this point cloud using the Poisson algorithm [16]. Afterwards we transpose the colors of the dense point cloud to the vertices of the Poisson mesh and automatically cut away the part without overlap with the dense point cloud. 


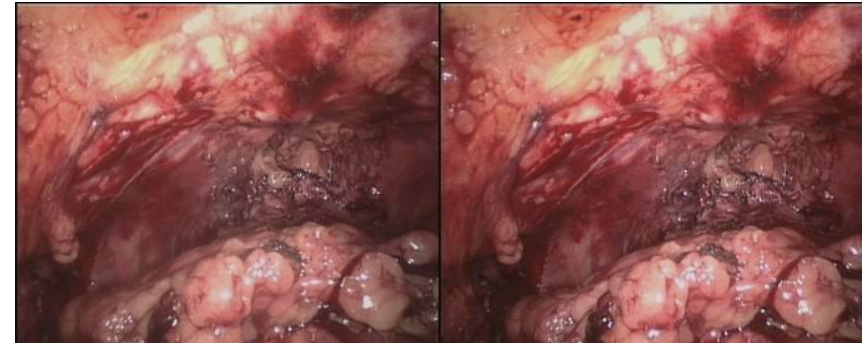

(a)

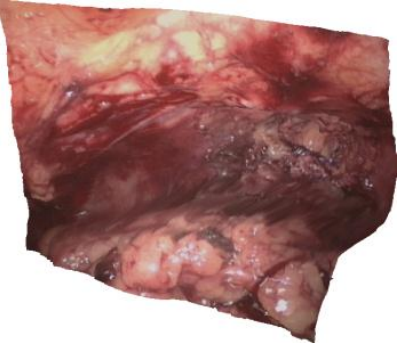

(d) (b)

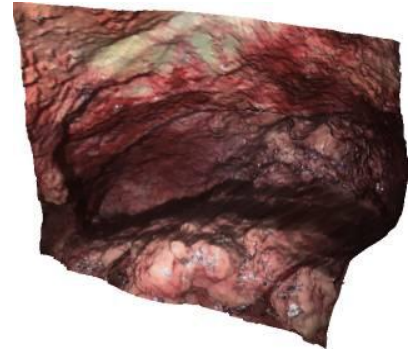

(e)

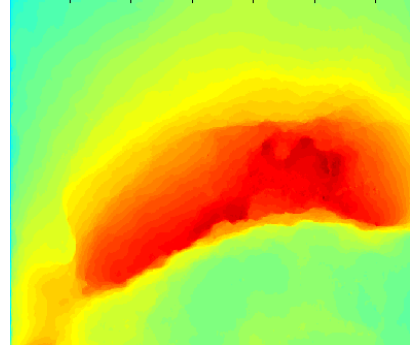

(c)

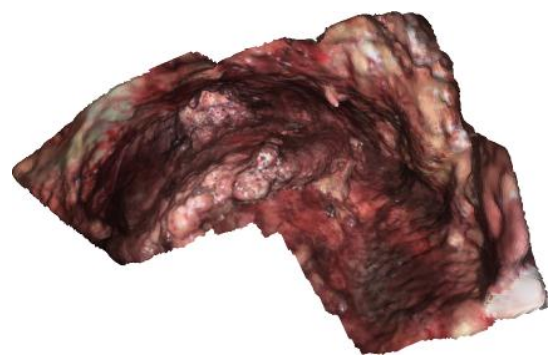

(f)

Figure 3. The 3D surface reconstruction shows a photorealistic environment. The upper row shows (a,b) combined original left and right image (c) resulting estimated depth map with the color green nearby and the color red far away. The bottom row shows $(\mathrm{d}, \mathrm{e})$ the resulting $3 \mathrm{D}$ model for one frame without and with shading and (f) the 3D panorama with shading.

\section{TEXTURE MAPPING}

This section describes the mapping of the textural color description on a smooth surface mesh. The geometrical shape model and the textural color description are separated to enable fast creation of shape models at acceptable quality while preserving all details that are visible in the input high-definition video stream.

Texture mapping can be used to improve the resolution of a colored mesh to the resolution of the original images (Figure 4). Texture mapping for a single image can be done by projection of the image onto the reconstructed surface. The starting point for our texture mapping method is a reconstructed surface and several camera images. The position of the camera images has been estimated, so that the image can be reprojected onto the surface. 3D reconstruction methods often produce an integrated surface mesh with less vertices than the original point cloud. This is to remove redundant data, outliers and noise, and to explicitly reduce the number of vertices in flat regions. Visual inspection of such a reconstructed surface without texture mapping appears blurry. By mapping the full resolution images on the surface, photorealism is preserved. Furthermore, texture mapping acceleration is extensively supported by almost all modern graphical hardware. So using it instead of increasing the number of 3D points (vertices) enables real-time 3D visualization of complex models.

For our reconstruction there are multiple images which have overlapping projections. Therefore, we need a method that selects the best image parts and maps those on the 3D surface. Afterwards some geometric and color artifacts remain at the borders of the different regions used. The main sources for these artifacts are:

1. An error in the reconstructed surface or an error in the ego-motion estimation;

2. Deformation of the surface (e.g. due to heartbeat and breathing induced motion);

3. Color and illumination variations in the images due to change in lighting and specular reflections.

In our research we have not yet tried to add compensations for distortions due to 1) and 2). We did, however, implement a method that is used to both i) select regions of images to be used for texture mapping, and ii) reduce the color and illumination variations. Each is discussed in the following subsections. 


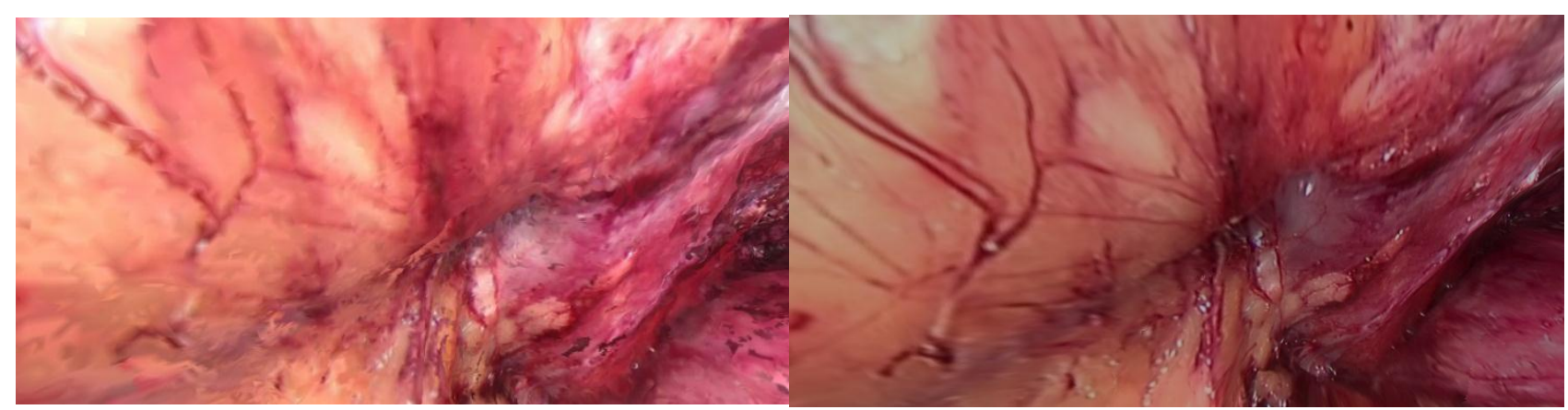

Figure 4. A Poisson surface reconstruction without texture mapping (left) and a surface with texture mapping (right).

Note the extra resolution given by the texture mapping.

\subsection{Texture mapping region selection}

The goal of texture mapping is to obtain a 3D visualization with the highest resolution and quality. For each 3D point we can calculate the best image to be used for texture mapping for a region near this $3 \mathrm{D}$ point. We do this by calculating for all sample points a score for each projection image based on:

1) The distance between the surface and the image

2) The angle between the viewing direction and the surface normal

3) The local sharpness of the image (we use [20] for sharpness estimation).

4) The distance from the projected point to the image boundary (near the image border the distortion and vignetting is higher)

For texture mapping, we make a selection of sample points $X_{i}$ on the 3D surface. These sample points can be chosen freely. In our system we have used both random points and evenly distributed points. For each point $X_{i}$ we can calculate the projected 2D point in camera $j$, we denote this point by $x_{\{i, j\}}$. Let $n_{i}$ be an estimated normal vector to the surface at $X_{i}$ and $\mathrm{v}_{\{\mathrm{i}, j\}}$ the direction from $X_{i}$ to $x_{\{i, j\}}$. We denote by $B(i, j)$ the sharpness of the image at $x_{\{i, j\}}$ as measured by the algorithm [20]. Let $\mathrm{R}$ be the radius of the image (in pixels) and $d_{i, j}$ be the distance from point $x_{\{i, j\}}$ to the border of the image. The scoring function used is:

$$
S C(i, j)=\alpha \operatorname{distance}\left(\mathrm{X}_{\mathrm{i}}, x_{\{i, j\}}\right) * \operatorname{dot}\left(n_{i}, v_{\{i, j\}}\right)+B(i, j) *\left(1-\beta \frac{d_{i, j}}{R}\right)
$$

The first term in the scoring function is the theoretical sharpness, assuming we have perfect imaging but a limited number of pixels. The second term is a more empirical term taking into account that the scene is not everywhere in focus and that the imaging is best at the center of the image. The variables $\alpha$ and $\beta$ are parameters that can be tuned to the dataset.

For each sample point on the surface, we then take the image with the best texture mapping score as the input image. We define texture mapping regions on the surface by calculating for each point on the surface the nearest sample point. By applying a smoothing operation to the regions we prevent fragmentation of the texture mapping regions. In particular we remove isolated regions (Figure 5). 


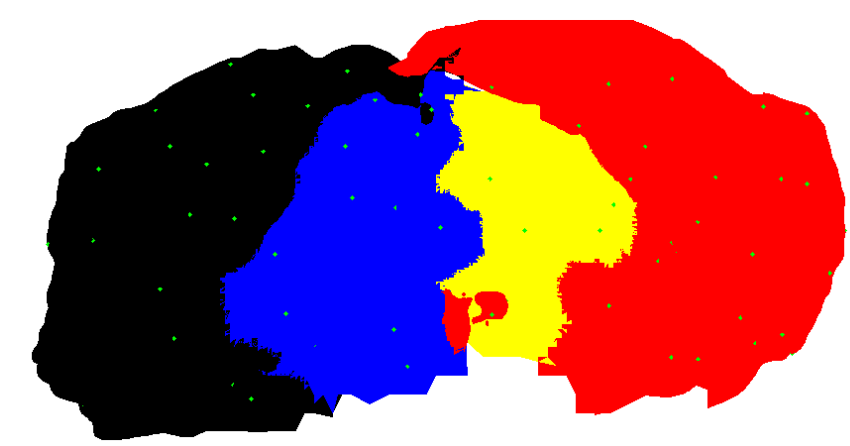

Figure 5. Surface with different regions (indicated by coloration) to be used for texture mapping. The image to be used for each sample point is determined by Equation (1). The regions are then calculated by using a distance map and removing isolated regions.

\subsection{Color correction}

Problems due to color and illumination variations in the images are caused by a moving light source that is fixed to the endoscope, or vignetting which makes the images darker near the boundary. The stitching of images without color correction results in a terrible 'Frankenstein'-effect (Figure 6).
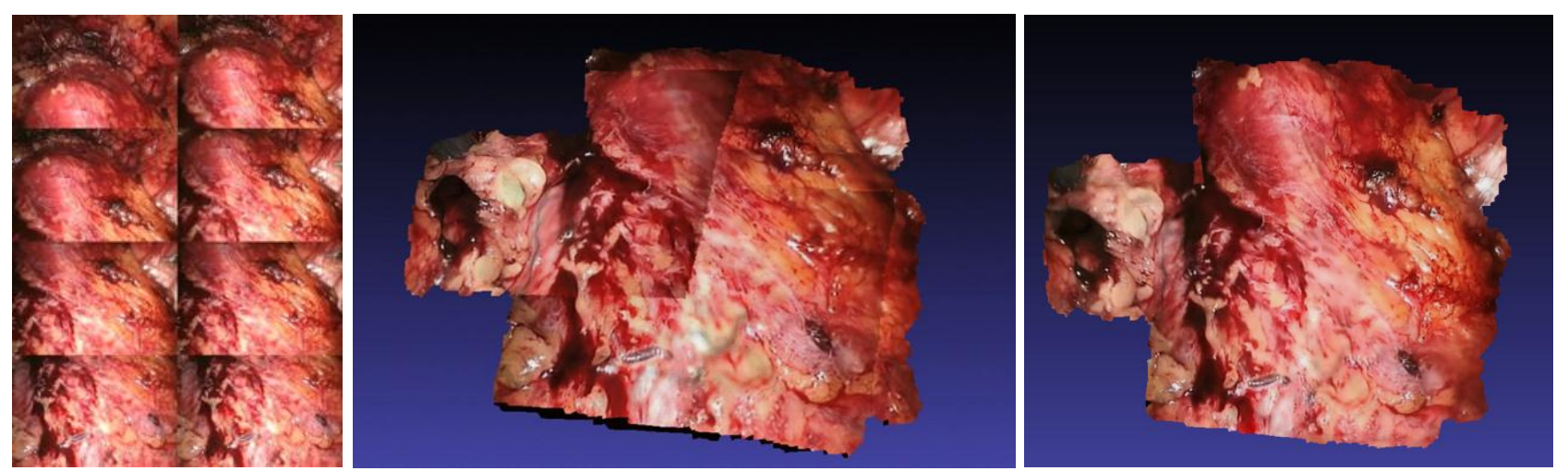

Figure 6. Texture mapping requires stitch and color correction to avoid a "Frankenstein"-effect. Input images (left), texture map without color correction (center), texture map with color correction.

Color corrections are created similar to the region selection, but instead of creating regions on the 3D surface, we create color correction regions in the 2D images.

For each sample point on the 3D surface and for all corresponding 2D projections we can sample the average color near this point. On the images we do this by taking the average color near a projected point in a small region, we have used a typical sampling distance of 8 pixels. On the 3D surface we sample a color by taking the average color of all nearby surface vertices. These nearby points can be efficiently calculated using a KNN structure [1].

For a single image there are many projected points and for each projection we can compare the sample color from the 3D point and the sample color from the projection. For each pair of a 3D surface point $X_{i}$ and a projected point in the image $x_{\{i, j\}}$ we can calculate a scaling of the color (either in RGB or HSV color space) that matches the colors. By scaling the intensity and saturation channels of the colors, we can calculate a scaling factor that makes the colors in the projected image similar to the sample color of the 3D point. This yields for each $2 \mathrm{D}$ projection a scaling factor for the color. We extrapolate the scaling factor to the entire image by using a distance transform and some smoothing operations.

An example of color correction is given in Figure 7. The image is a single frame from the video stream of a stereo endoscope. Due to the position of light source with respect to the stereo endoscope, the left video channel has a dark band in the right (and the right video channel has a dark band on the left). Note that in the color corrected image, the dark border on the right of the image has been corrected. 


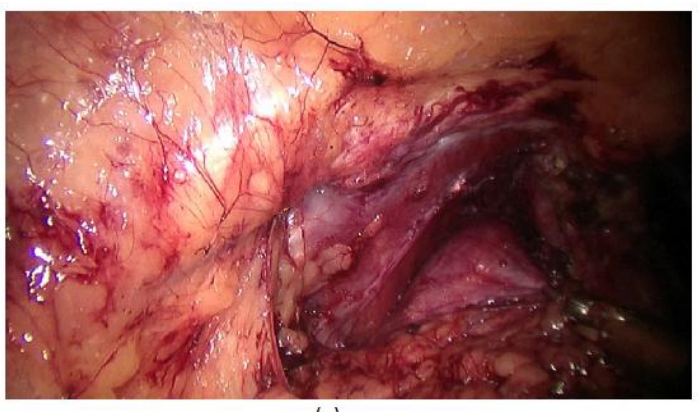

(a)

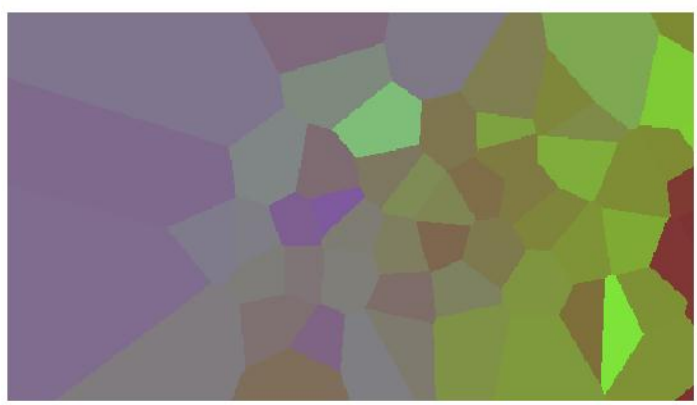

(c)

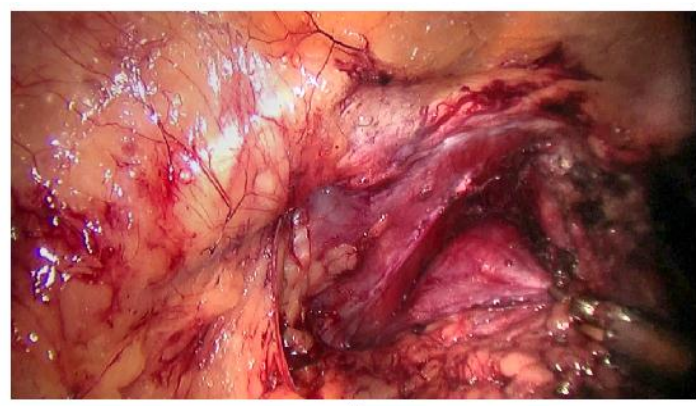

(b)

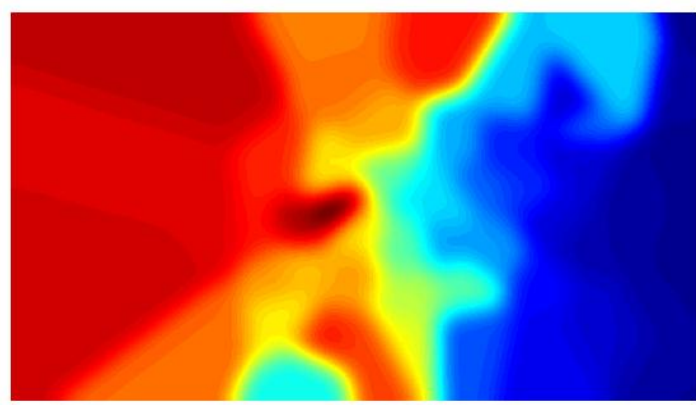

(d)

Figure 7. Color correction applied to a single image. (a) Original input image, (b) Color corrected image, (c) color correction regions, (d) scaling in value channel. Because the regions and correction factors are determined using the sample points on the $3 \mathrm{D}$ surface, the corrections to the individual images are consistent with all the other images.

Using the scaling factor that is calculated on the entire image we can calculate color corrected images. All images have been corrected to match the colors on the reconstructed surface. This means that we have created a global color correction, and the colors of the images will agree on the borders of the texture mapping.

So far we have not dealt with non-rigid surfaces or reconstruction errors. A possible method to do this is to calculate deformations necessary in order to make the different images agree. Calculation of the deformations can be done using methods such as optic flow. Then the method of sampling points on the reconstructed surface can again be used to calculate deformations of the images that are globally consistent (and not only locally).

\section{EXPERIMENTS AND RESULTS}

\subsection{Experimental setup}

For the experiment, we recorded streaming video of a radical prostatectomy that was performed with a first generation DaVinci Robot of Intuitive Surgical in Canisius Wilhelmina Hospital (CWZ) in Nijmegen, The Netherlands. This DaVinci system generates stereoscopic (left and right channel) S-Video, which was digitized using two synchronized Pleora iPORT engines (PT1000-ANL-1-6-V2-E) and captured with a DELL XPS laptop with RAID hard disk system, 2x GB-LAN (1 connector and 1 express card). Newer generations of the DaVinci Robot generated stereoscopic HD-SDI, which was recorded with two synchronized Panasonic P2 recorders (AG-HPD24E).

Tools used for visualization are a viewer developed by TNO based on OpenSceneGraph [26]. This viewer can show points clouds, meshes and perform region-based texture mapping. The viewer can display the reconstructions on screens with and without 3D.

Live projection [35] of video streams on a 3D HDTV is possible, e.g. with Panasonic (TX-P50VT20E) or Sony (LMD2451MT). The Sony product is developed for medical applications and uses passive (polarized) glasses (Figure 9). 


\subsection{Results}

From the recorded video stream, we are able to generate photorealistic 3D panoramic environments from a mono- or stereoscopic video stream (Figure 8).

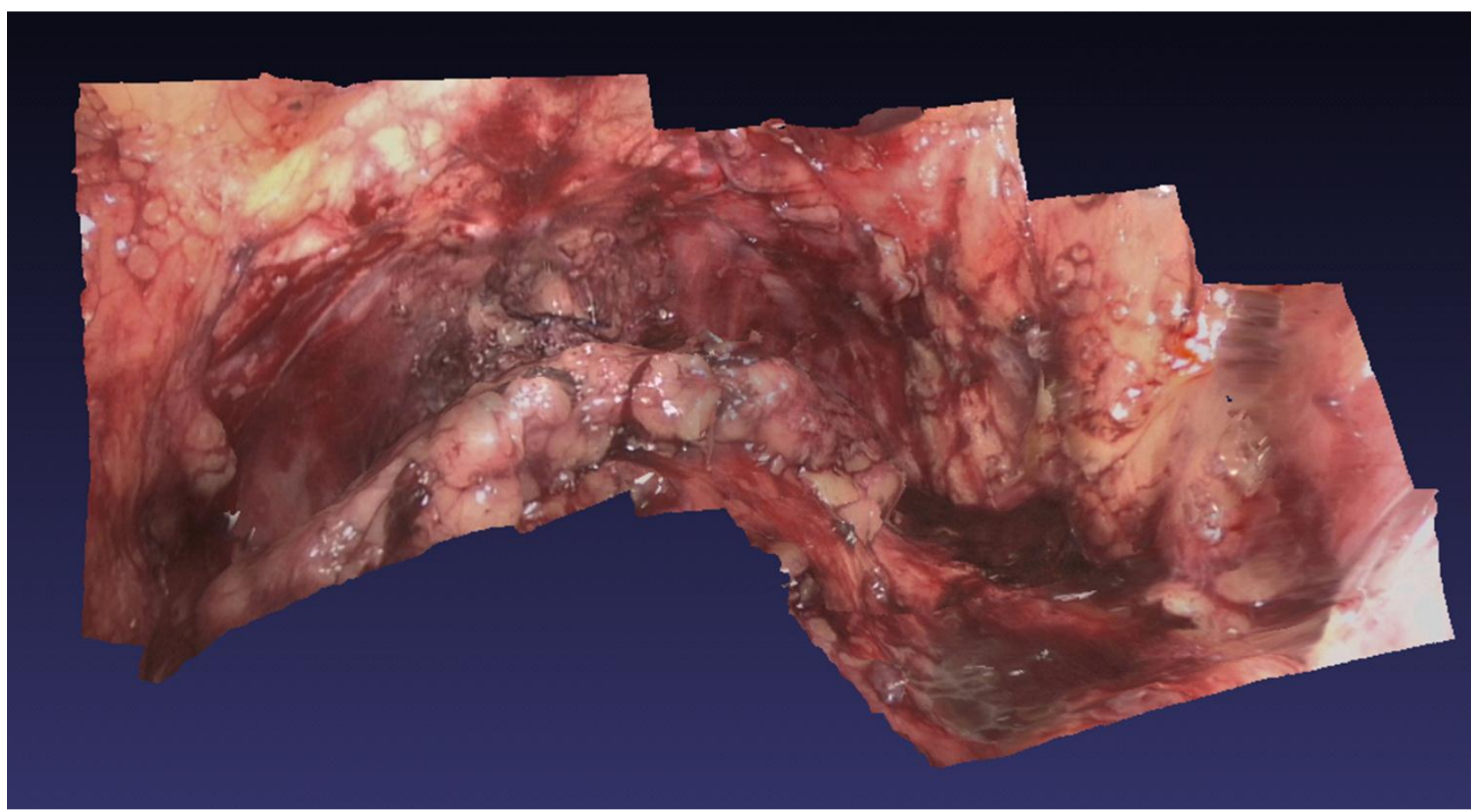

Figure 8 . The dense 3D reconstruction shows a photorealistic environment.
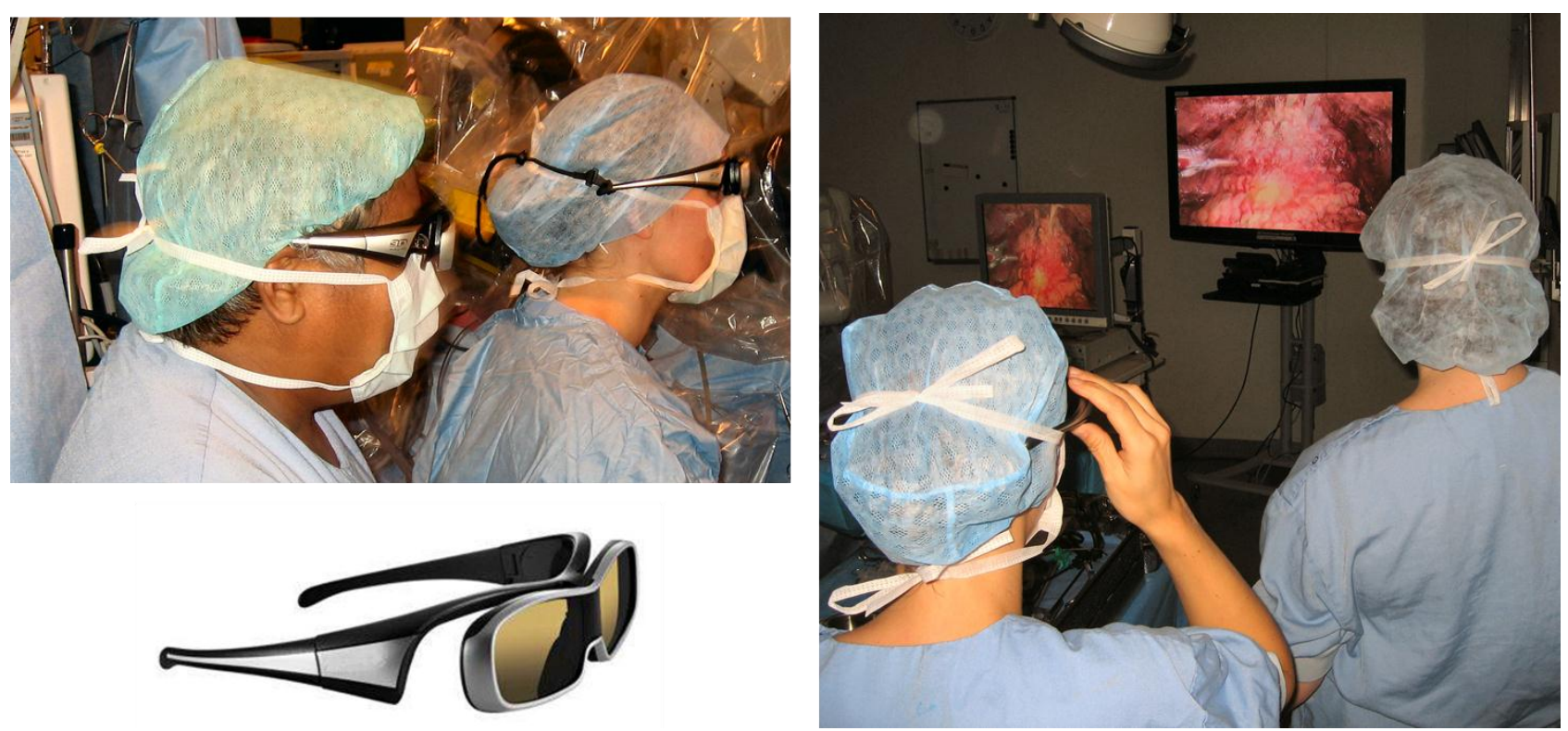

Figure 9. Live 3D view, recording, and playback on a 3D television in the operation theatre. 


\section{CONCLUSIONS}

We described a novel technology that can reconstruct 3D panoramas from video streams of body cavities explored by endoscope. Our methods are compatible with any mono- or stereoscopic endoscope and provides a much wider cumulative overview than single endoscopic image frames can offer. The technology is an add-on to existing endoscopy systems. It does not need position tracking, additional markers or expensive (MR or CT) pre-scans, because it is purely based on information that is extracted from the endoscopic images.

We demonstrate photorealistic 3D environments generated from single channel (mono) and dual channel (stereo) laparoscopic video data. To assist in navigation, the method can visualize position and orientation of the endoscope relative to the environment as viewed from a perspective outside the endoscope. The method also allows accurate measurement of distances and sizes. The resulting 3D reconstructions can be directly applied in endoscopic simulators and e-learning. Extended to real-time processing, the method looks promising for tele-surgery or other remote visionguided tasks requiring spatial visualization and complex navigation.

\section{ACKNOWLEDGEMENT}

The work in this paper was financially supported by SenterNovem (currently AgentschapNL) in the high-tech top project IGIT4Health (HTT09011). Streaming video data courtesy of the Canisius-Wilhelmina Hospital (CWZ) in Nijmegen, The Netherlands.

\section{REFERENCES}

[1] Arya, S., Mount, D., Netanyahu, N., Silverman, R., Wu, A., “An optimal algorithm for approximate nearest neighbor searching in fixed dimensions", Journal of ACM 45(6), (1998).

[2] Basten, J.P., Beerlage, H., Tjiam, I., [Aanbevelingen laparoscopie in the urologie], Verhagen, Veldhoven The Netherlands, (2010).

[3] Bouarfa, L., Akman, O., Schneider, A., Jonker, P.P., Dankelman, J., "In-vivo real-time tracking of surgical instruments in endoscopic video", Minim. Invasive Therapy Allied Techn., (2011).

[4] Broeders, I.A.M.J., Kalisingh, S.S., [Handbook endoscopic surgery], Bohn Stafleu van Loghum, Houten The Netherlands, (2009).

[5] Budde, R.P.J., Meijer, R., Bakker, P.F.A., Borst, C., Grundeman, P.F., "Endoscopic Localization and Assessment of Coronary Arteries by 13 MHz Epicardial Ultrasound", Annals of Thoracic Surgery 77, (2004).

[6] Chan, M., Lin, W., Zhou, C., Qu, J.Y., "Miniaturized three-dimensional endoscopic imaging system based on active stereovision", Applied Optics 42(10), 1888-1898 (2003).

[7] Davison, A.J., "Real-time simultaneous localization and mapping with a single camera", Proc. ICCV, (2003).

[8] Dubbelman, G., van der Mark, W., Groen, F.C.A., "Accurate and robust ego-motion estimation using expectation maximization”, IEEE Int. Conf. Intel. Robots and Systems, 3914-3920 (2008).

[9] Durrani, A.F., Preminger, G.M., “Three-dimensional video imaging for endoscopic surgery", Computers in Biology and Medicine 25(2), 237-247 (1995).

[10]Esteban, I., Dijk, J., Groen, F.C.A., "FIT3D toolbox: multiple view geometry and 3D reconstruction for Matlab", Proc. SPIE 7835, (2010).

[11] Furukawa, Y., Ponce, J., “Accurate dense and robust multi-view stereopsis”, IEEE CVPR, (2007).

[12] Gronemeyer, D.H., Seibel, R.M., Schmidt, A., Melzer, A., Dell, M., "Two- and three-dimensional imaging for interventional MRI and CT guidance", Studies in Health Technologies and Informatics 29, 62-76 (1996).

[13] Iersel, M. van, Veerman, H., Mark, W. van der, "Modeling a crime scene in 3D and adding thermal information", Proc. SPIE 7481, (2009).

[14] Intuitive Surgical - Da Vinci Surgical System: intuitivesurgical.com/products/davinci_surgical_system 
[15] Kanatani, K., [Statistical optimization for geometry computation: theory and practice], Dover Publications, Mineola NY USA, (2005).

[16] Kazhdan, M., Bolitho, M., Hoppe, H., "Poisson surface reconstruction”, Proc. Eurographics Symp. Geom. Processing, 61-70 (2006).

[17] Klein, G., Murray, D.W., "Parallel tracking and mapping for small AR workspaces", Proc. Int. Symp. Mixed and Augmented Reality, (2007).

[18] Konen, W., Scholz, M., Tombrock, S., "The VN-project: endoscopic image processing for neurosurgery", Computer Aided Surgery 3, 1-6 (1998).

[19] Konolige, K., "Projected texture stereo", IEEE Conf. Robotics and Automation, (2010).

[20] Kristan, M., Pers, J., Perse, M., Kovacic, S., “A Bayes-spectral-entropy-based measure of camera focus using a discrete cosine transform", Pattern Recognition Letters, 27(13), 1419-1580, (2006).

[21] van der Mark, W., Gavrila, D.M., "Real-time dense stereo for intelligent vehicles", IEEE Trans. Intelligent Transport Systems 7(1), 38-50 (2006).

[22] van der Mark, W, Burghouts, G, den Dekker, E, ten Kate, T, Schavemaker, J, "3-D scene reconstruction with a handheld stereo camera", COGnitive systems with Interactive Sensors (COGIS), (2007).

[23] Microsoft Kinect: www.xbox.com/Kinect

[24] Newcombe, R.A., Davison, A.J., "Live dense reconstruction with a single moving camera", Proc. IEEE CVPR, 1498 - 1505 (2010).

[25] Newcombe, R.A., Lovegrove, S.J., Davison, A.J., "DTAM: Dense tracking and mapping in real-time", Proc. IEEE ICIP, (2011).

[26] OpenScenceGraph: openscenegraph.org

[27] Personal space technologies: ps-tech.com

[28] Reeff, M., Gerhard, F., Cattin, P., Szekely, G., "Mosaicing of endoscopic placenta images", Lecture Notes in Informatics P-93, 467-474 (2006).

[29] Ringbeck, T., “A 3D time-of-flight camera for object detection”, Optical 3-D Measurement Techniques, (2007).

[30] Snavely, N., Seitz, S.M., Szeliski, R., "Photo tourism: exploring image collections in 3D", ACM Trans. Graphics 25(3), 835-846 (2006).

[31] Strecha, C., von Hansen, W., Van Gool, L., Fua, P., Thoennessen, U., “On benchmarking camera calibration and multi-view stereo for high resolution imagery", IEEE CVPR, (2008).

[32] Tabaee, A., Anand, V.K., Fraser, J.F., Brown, S.M., Singh, A., Schwartz, "Three-dimensional endoscopic pituitary surgergy", Neurosurgery 64, 288-295, (2009).

[33] Thormaehlen, T., Broszio, H., Meier, P., “Three-dimensional endoscopy”, Falk Symposium - Medical Imaging in Gastroenterology and Hepatology 124, 199-212 (2002).

[34] Wedel, A., Pock, T., Zach, C., Bischof, H., Cremers, D., “An improved algorithm for TV-L1 optical flow”, LNCS 5064, 23-45 (2009).

[35] Wieringa, F.P., Bouma, H., Beerlage, H.P., "Driedimensionaal meekijken met de chirurg; Innovaties in 3Dbeeldtechniek", OK Operationeel, 13-23 (2012).

[36] Wieringa, F.P., Bouma, H., Eendebak, P., van Basten, J.P., Beerlage, H.P., Smits, G., Schols, R., Stassen, L.P.S., "Visualization in the operation room; From looking good to looking better", Year congress Dutch assoc. endoscopic surgery NVEC, (2012).

[37] Yao, K., Matsui, T., Furukawa, H., Yao., T., Sakurai, T., Mitsuyasu, T., "A new stereoscopic endoscopy system: accurate 3-dimensional measurement in-vitro and in-vivo with distortion correction function", Gastrointestinal Endoscopy 55(3), 412-420 (2002). 